\title{
ENERGY EFFICIENT TOPOLOGIES For WIRELESS SENSOR NETWORKS
}

\author{
Gurwinder Kaur $^{1}$ and Rachit Mohan Garg ${ }^{2}$ \\ ${ }^{1,2}$ Department of Education and Research, Infosys Limited, Mysore, India \\ gurwinder.kaurmatharu@gmail.com, rachit.mohan.garg@gmail.com
}

\begin{abstract}
A wireless network is a group of spatially dispersed specialized transducers with a sensing, computing and communication infrastructure which intends to give them the ability to sense and record various conditions in a monitored environment. They act as interfaces between real and virtual worlds. They are the most rapidly developing information technologies in today's world due to their vast range of applications. This paper gives an overview of wireless sensor networks and provides a scenario based comparison for energy efficiency between different topologies used in these networks.
\end{abstract}

\section{KEYWORDS}

Wireless Sensor Networks, topologies, energy efficiency, lifetime, power consumption.

\section{INTRODUCTION}

Wireless sensor networks are the networks which are formed with a group of devices equipped with sensors distributed in space. These devices are used to measure and record various environmental conditions in diverse locations. A sensor network is made of sensor nodes where each sensor node is equipped with a radio transceiver along with an antenna, a microcontroller, an interfacing electronic circuit and an energy source (usually a battery). Each sensor node can be imagined as a small computer consisting of a processing unit and a limited amount of computational power and memory.

The main difference between the Wireless Sensor Networks (WSNs) and adhoc networks is that for WSNs, the main functions are monitoring and collecting the data whereas for the adhoc networks, the main focus is on communication aspects.

A number of efforts have been made by industry and academia to develop and deploy WSN so that it can satisfy a variety of applications. But the biggest limitation of WSN devices is their power constraint which affects their lifetime and area of coverage. Proper area coverage ensures that the complete monitored area can be represented by the data collected from the sensors while the lifetime refers to the operating time of the WSN for collecting data from the entire network domain.

In a given network domain, different deployment strategies of sensors often lead to different network coverage and lifetime values, which are very important design criteria for any WSN. Placing sensors in a controllable manner to achieve maximum network lifetime is the most effective approach of sensor deployment. But this approach may prove to be technically or practically non-feasible especially for large WSNs. Also, some locations of the network domain may not be reachable to the network planners due to geographical constraints.

DOI : $10.5121 /$ ijdps.2012.3516 


\section{Parameters For Measuring The Effectiveness Of a TOPOLOGY:}

2.1 Range and coverage: Range and coverage are probably the most obvious requirements for a WSN starting from the node to node range at a given transmission power / antenna gain and data rate. The main factors affecting range of a wireless network are the quality of physical layer and the efficiency of data transmission through the network. The coverage requirements are elimination of dead spots in the network and the extent of coverage area in range, both of which are closely related to range.

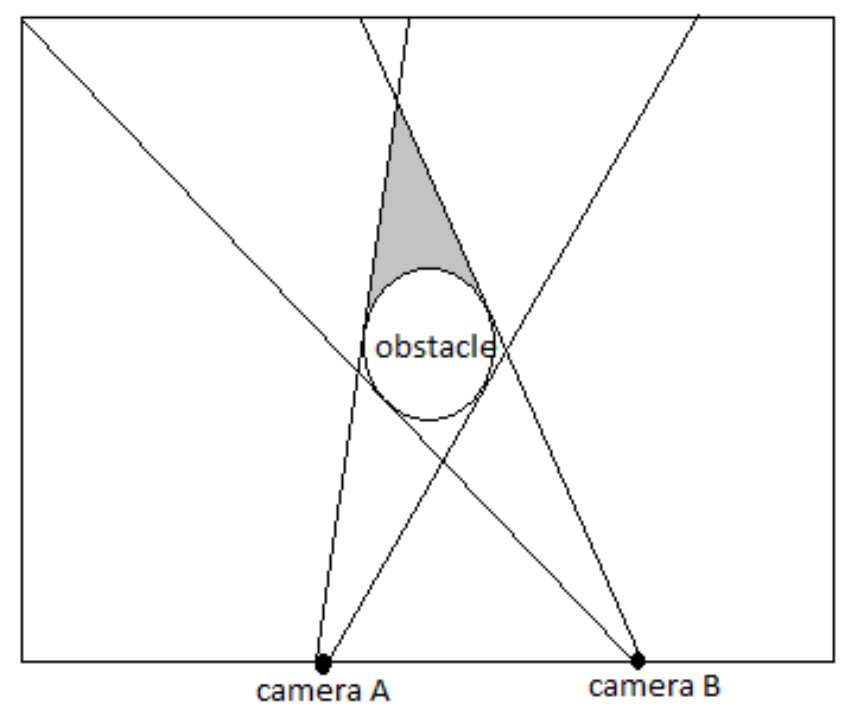

Fig. 1: An obstacle reduces the coverage of two cameras directed at different areas

2.2 Scalability: Scalability is the property of being able to cope up with network cells as small as a few nodes to cells of thousands or even tens of thousands of nodes as well as increasing the size of existing network by order of magnitude without employing expensive cellular communication or other long range solutions.

2.3 Expected Transmission Count (ETX): It accounts for data loss due to medium access contention and environmental hazards and considers the number of transmissions needed to successfully transmit a packet over a link.

2.4 Hop Count: Hop count is the most commonly used metric in wireless multi-hop networks. The path having the minimum number of links between a given source and the destination node is selected.

2.5 Power consumption/Network Longevity: In most of the published communication protocols for WSNs, network lifetime extension has been mentioned as an important optimization objective. The position of nodes can affect the network lifetime significantly. For example, a nonuniform node distribution in a given area may lead to bottlenecks and unbalanced traffic. On the other hand, uniform distribution of nodes in a network may result in depletion of energy of nodes 
that are close to the base station at a rate higher than the other nodes, which in turn will shorten the network lifetime.

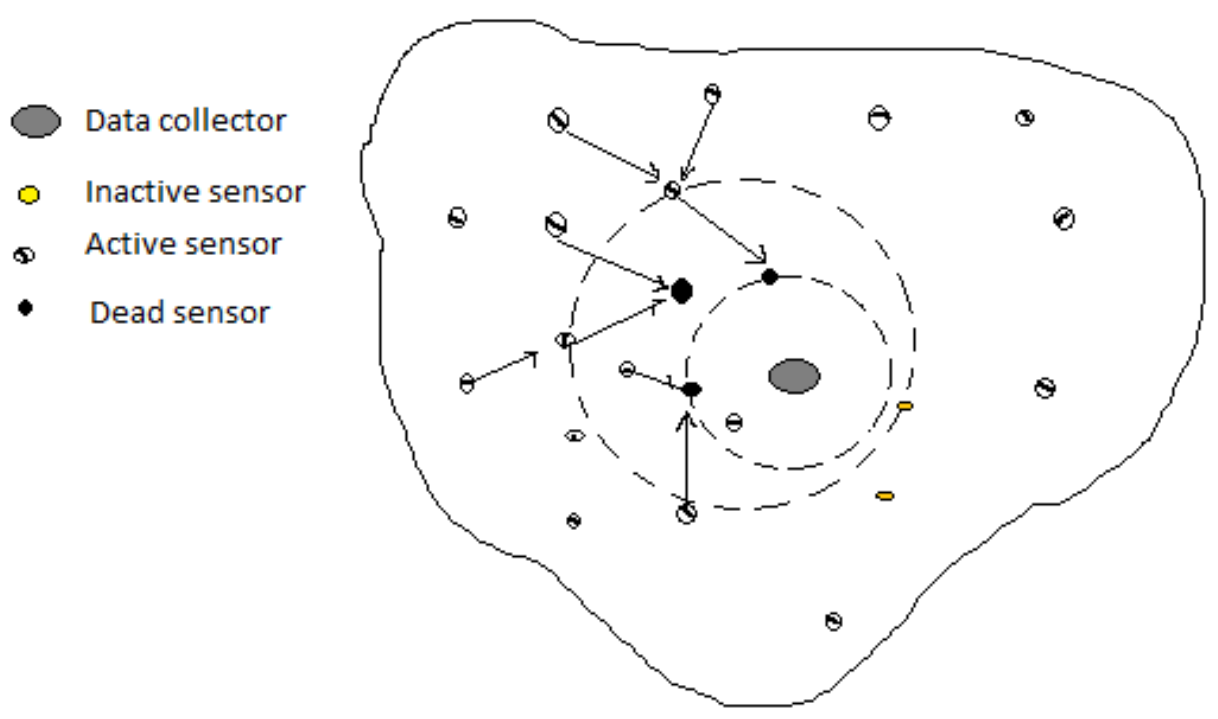

Fig 2: Nodes closer to the base station/ data collector die quickly due to more traffic.

\section{TOPOLOGIES FOR A WiRELESS SENSOR NETWORK}

The network structure of wireless network is not limited to one design. While designing the network, the developer has several choices of topologies for configuring the network. Following are the different topologies:

3.1 Single hop star topology: Single hop star topology is the simplest WSN topology. In this topology, every node communicates directly with the gateway or the data collector. Due to minimum networking concerns, this topology simplifies the network wherever it is realizable. However, due to its design only, the biggest limitation it possesses is the problem of scalability. The nodes that are at a large distance from the gateway will have poor quality connections with the gateway. Thus, this topology is good to be used only when the number of nodes in the network is very small and the coverage area does not extend beyond the radio transmission range of around 30 meters in a building. 
International Journal of Distributed and Parallel Systems (IJDPS) Vol.3, No.5, September 2012

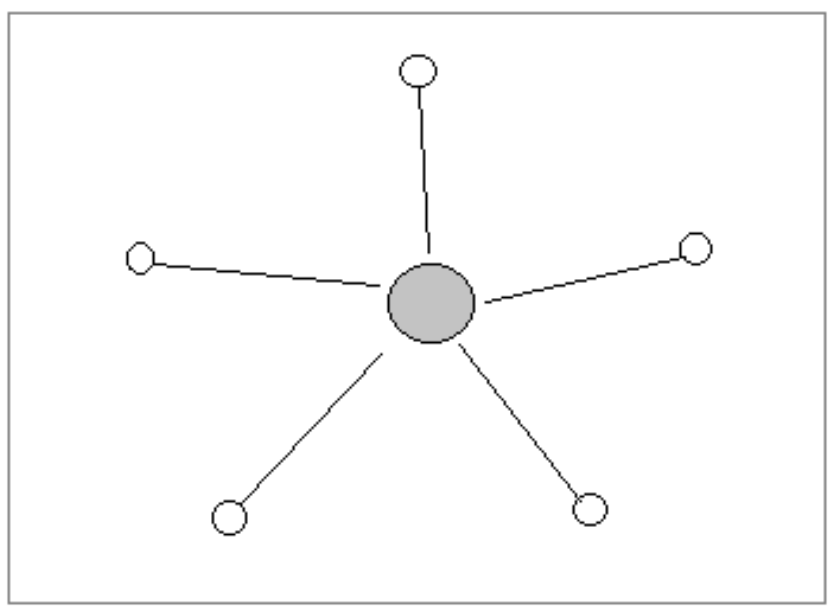

Fig 3: Single hop star topology

3.2 Multi hop mesh and grid topology: For covering large are, multi hop network is necessary. In this topology, the signal goes from one sensor to the other until it reaches the gateway. Here, the route of the signal is determined by a particular routing protocol. Depending upon whether the network is random or structured, it can look like the below figures:

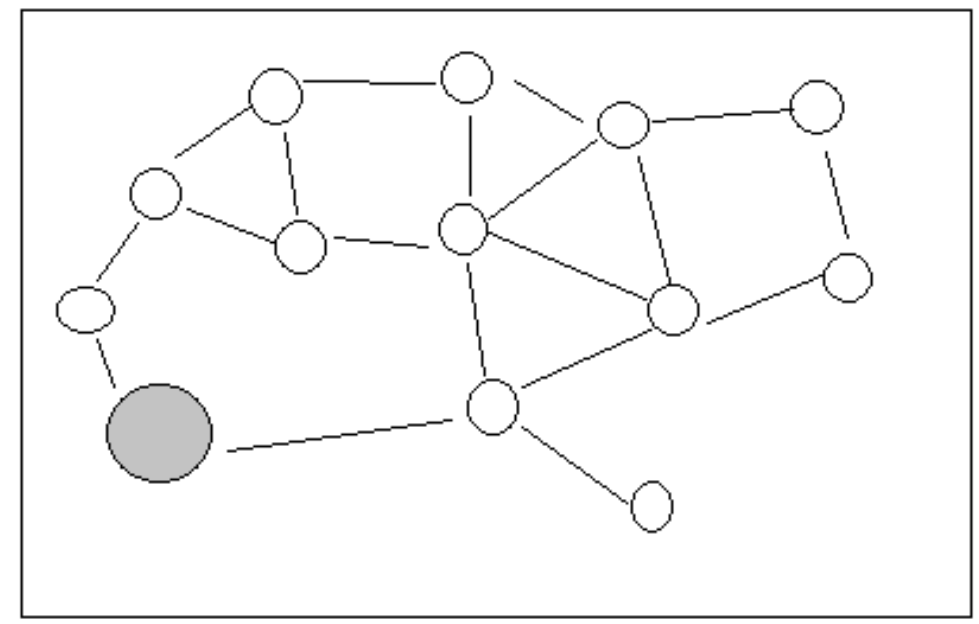

Fig.4: multi hop star topology (random) 


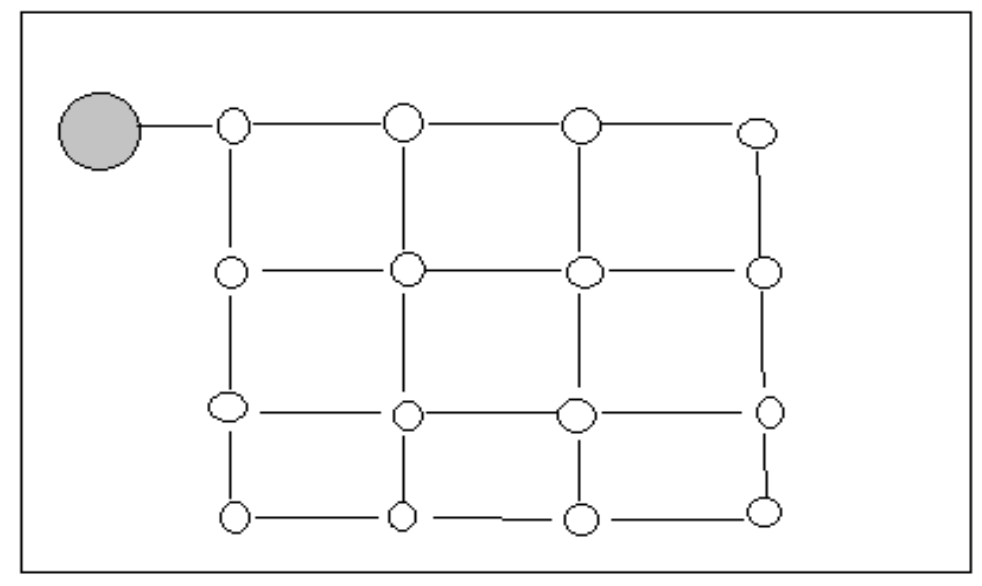

Fig.5: multi hop star topology (structured)

The properties of self-configuration, self-healing and scaling provide flexibility to the mesh network.

3.3 Two-tier hierarchical cluster topology: This is the most common architecture for larger WSNs. In this topology, nodes within a specific region send their data to a local cluster head. In turn all such cluster heads from different regions send their collected data to the gateway. This network can be interlaced further also i.e. the cluster head of tier 2 can send the data to the cluster head of another network which can further send the data to the gateway. The biggest advantage of this topology is that it divides the whole network into a number of small zones within which routing of signals can be done locally. The cluster heads can be designed to be more powerful in terms of computation/communication. In addition to it, the nodes can also be connected through a wire, which increases the transmission speed as well as reliability of the network.

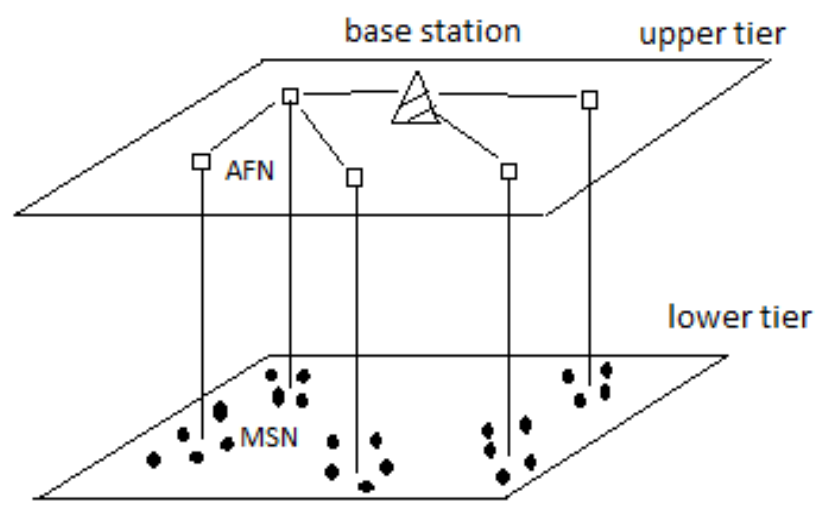

Fig.6: Two tier hierarchical structure. 


\section{COMPARISON OF DIFFERENT TOPOLOGIES:}

\begin{tabular}{|l|l|l|l|}
\hline Topology & Node Degree & Network diameter & Network size \\
\hline Linear Array & 2 & N-1 & N nodes \\
\hline Ring & 2 & Floor(N/2) & N nodes \\
\hline Completely connected & $\mathrm{N}-1$ & 1 & N nodes \\
\hline Binary tree & 3 & $2(\mathrm{~h}-1)$ & $\mathrm{h}=$ ceil(log2N) \\
\hline Star & $\mathrm{N}-1$ & 2 & N nodes \\
\hline 2-D mesh & 4 & $2(\mathrm{r}-1)$ & r*r mesh where $\mathrm{r}=\sqrt{ } \mathrm{N}$ \\
\hline
\end{tabular}

Here we are considering a scenario where the sensors are uniformly scattered on a straight line in the interval $[-D ; D]$ and the fusion center is $h$ meters away from the midpoint of the line $(h>D)$. The distance between the sensor ' $i$ ' and sensor ' $n$ ' is denoted by $d$ i,n.

Here in this scenario,a comparison of three different categories of topologies is done on the basis of energy efficiency \& lifetime.Below are the categories of topologies:

4.1 Cluster based Sensor Network:Cluster based sensor network consists of two types of sensors. Sensor $\beta$ acts as cluster head and the rest of the sensors play the role of cluster members. The data to be transmitted from these cluster members is constrained to M bits. Each member of the cluster quantizes its observed data to $\mathrm{M}$ bits and transmits this quantized data to the cluster head. The cluster head receives the quantized data from the other sensors and performs source extraction based on its own observation and the data received.

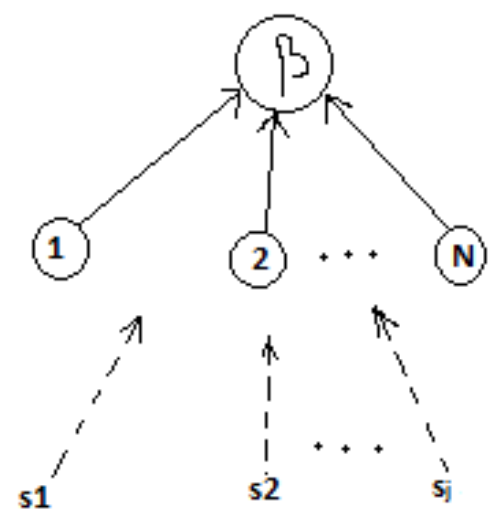

Fig.7: cluster based sensor network

4.1.1 Impact on energy efficiency:The task is to extract a signal frame with length $L$ in each round at the cluster head. In the cluster based sensor network, the total energy consumed for communications is calculated as below:

$$
\mathrm{E}_{\text {comm }}^{(1)}=2(\mathrm{~N}-1) \mathrm{MLE}_{\text {elec }}+\mathrm{ML} \epsilon_{\mathrm{fs}} \sum=0 \quad{ }_{\mathrm{i}, \beta}^{2}, \mathrm{i} \neq \beta
$$

Where $\mathrm{M}$ is the number of quantization bits, $\mathrm{L}$ is the length of a signal frame, $\mathrm{E}_{\text {elec }}$ is the energy consumed for running the electronic circuits and $\epsilon_{\mathrm{fs}}$ is an amplifier energy factor. The computation 
mainly consists of three parts: quantization, prewhitening and iteration of the fast fixed-point algorithm which are approximated by $\mathrm{LE}_{\mathrm{c}}, \mathrm{N}^{2} \mathrm{LE}_{\mathrm{c}}$ and $\mathrm{LE}_{\mathrm{c}}$ respectively, where $\mathrm{E}_{\mathrm{c}}$ is the

unit energy for computation. Therefore, the total energy consumed for computation in the cluster based sensor network is :

$$
\mathrm{E}_{\text {comp }}^{(1)}=\left[(\mathrm{N}-1) \mathrm{L}+\mathrm{N}^{2} \mathrm{~L}+\mathrm{L}^{2}\right] \mathrm{E}_{\mathrm{c}}
$$

The total energy consumed for sensing is $E_{s e n}=N_{L E}$, where $E_{s}$ is the unit of energy for sensing one sample point. This part of energy consumption is the same for all the sensor networks. Hence, the total energy consumption in the cluster based sensor network for extracting a signal frame is:

$$
\mathrm{E}_{\text {total }}^{(1)}=\mathrm{E}^{(1)} \text { comm }+\mathrm{E}^{(1)} \text { comp }+\mathrm{E}_{\text {sen }}
$$

4.1.2 Impact on lifetime: Lifetime $\mathrm{T}$ is defined as the time from the round that the sensor network starts working to the round that only $\mathrm{J}$ surviving sensors can afford the energy to extract a signal frame. In the cluster-based sensor network, the energy budgets of the cluster head and the cluster members are $\mathrm{E}_{1}$ and $\mathrm{E}_{2}$ respectively. In the cluster-based sensor network, the energy consumption of each cluster member and the cluster head is $\mathrm{E}_{\mathrm{c}}+\mathrm{LE}$ elec $+\mathrm{MLE}$

$$
+\mathrm{ML} \in \mathrm{d}^{2}
$$

(N-1) $\mathrm{MLE}_{\mathrm{elec}}+\left(\mathrm{N}^{2} \mathrm{~L}+\mathrm{L}^{2}\right) \mathrm{E}_{\mathrm{c}}$ respectively. Mathematically,

$$
\mathrm{TE}_{\mathrm{m}, \mathrm{J}} \leq \mathrm{E}_{2}<(\mathrm{T}+1) \mathrm{E}_{\mathrm{m}, \mathrm{J}}
$$

In the cluster based sensor network, the energy consumption of the cluster members is arranged in descending order. Mathematically,

$$
\begin{gathered}
\mathrm{TE}_{\mathrm{h}} \leq \mathrm{E}_{1}<\underset{1}{(\mathrm{~T}+1) \mathrm{E}_{\mathrm{h}},}, \mathrm{E}_{2} \geq \mathrm{TE}_{\mathrm{m}, \mathrm{J}-} \\
\text { or } \\
\mathrm{TE}_{\mathrm{m}, \mathrm{J}-1} \leq \mathrm{E}_{2}<(\mathrm{T}+1) \mathrm{E}_{\mathrm{m}, \mathrm{J}-1}, \mathrm{E}_{1} \geq \mathrm{TE}_{\mathrm{h} .}
\end{gathered}
$$

The greater $\mathrm{T}$ derived from these two inequalities is the lifetime.

4.2 Sensor network with a fusion center: Here, the fusion center performs the operation of source extraction based on the received quantized data from the sensors.

4.2.1 Impact on energy efficiency:The equations below show the energy required in communication and computation in Sensoe Network with fusion center.

$$
\begin{aligned}
& \mathrm{E}^{(2)}{ }_{\text {comm }}=\mathrm{NMLE}_{\text {elec }}+\mathrm{ML} \epsilon_{\mathrm{mp}} \Sigma_{-} \quad 1 \mathrm{i}, \mathrm{f} \\
& \mathrm{E}_{\text {comp }}^{(2)}=\mathrm{NLE}_{\mathrm{c}}
\end{aligned}
$$

Where $\epsilon_{\mathrm{mp}}$ is another amplifier energy factor, $\mathrm{d}_{\mathrm{i}, \mathrm{f}}$ is the distance from sensor ' $\mathrm{i}$ ' to the fusion center.

4.2.2 Impact on lifetime: In the sensor network with a fusion center, the energy consumption of each sensor is:

$$
\mathrm{E}_{\mathrm{sen}}+\mathrm{LE}_{\mathrm{c}}+\mathrm{MLE}_{\text {elec }}+\mathrm{ML} \epsilon_{\mathrm{mp}} \mathrm{d}_{\mathrm{i}, \mathrm{f}}^{4}
$$

The sensors that are nearest to the midpoint of the line consume least energy for broadcasting and are selected for performing iterations. $\mathrm{T}$ is defined as the time from the round that the sensor 
network starts working to the round that only $\theta-1$ surviving sensors in the selected sensor set can afford the energy to extract a signal frame. Mathematically,

$$
\mathrm{TE}_{\mathrm{m}, \theta-1} \leq \mathrm{E}_{0}<(\mathrm{T}+1) \mathrm{E}_{\mathrm{m}, \theta-1}
$$

4.3 Concatenated sensor network:Source extraction is performed by the sensors in the concatenated network.

4.3.1 Impact on energy efficiency:The equations below show the energy required in communication and computation in concatenated sensor network.

$$
\begin{aligned}
& \mathrm{E}^{(3)}{ }_{\text {comm }}=[2 \mathrm{~N}(\mathrm{~N}-1) \mathrm{ML}+8 \mathrm{~J}(\theta-1)] \mathrm{E}_{\text {elec }} \\
& +\mathrm{ML} \epsilon_{\mathrm{fs}} \sum_{=1} \quad \sum=1 \quad{ }_{\mathrm{i}, \mathrm{n}}^{2}, \mathrm{n} \neq \mathrm{i} \\
& +4 \mathrm{~J}_{\mathrm{fs}_{\mathrm{s}}} \sum^{-}=12_{\phi, \phi+1}^{2} \\
& \mathrm{E}_{\text {comp }}^{(3)}=\left(\mathrm{NL}+\theta \mathrm{N}^{2} \mathrm{~L}+\mathrm{L}^{2}\right) \mathrm{E}_{\mathrm{c}}
\end{aligned}
$$

$\theta$ is the number of iteration steps in the concatenated sensor network and the sensors indexed by 1 to $\theta$ are those selected for performing the iteration. The selection criterion is to minimize the energy consumed for broadcasting the quantization bits and propagating $\mathrm{w}$ relating to distances. The latter is approximated as that consumed for transmitting a 4-bit message. To extract a signal frame successfully, the number of sensors in the concatenated sensor network must satisfy $\mathrm{N}>=\theta$.

4.3.2 Impact on lifetime:In the concatenated sensor network, the energy consumption of the sensors is one of the following:

a. $\mathrm{E}_{\mathrm{sen}}+\left(\mathrm{L}+\mathrm{N}^{2} \mathrm{~L}+\mathrm{L}^{2} / \theta\right) \mathrm{E}_{\mathrm{c}}+[2(\mathrm{~N}-1) \mathrm{ML}+8 \mathrm{~J}]$

$\mathrm{E}_{\text {elec }}+\mathrm{ML} \epsilon_{\mathrm{fs}} \sum=1 \quad{ }_{\phi, \mathrm{n}}^{2}+4 \mathrm{~J} \epsilon_{\mathrm{fs}} \mathrm{d}_{\phi, \phi+1}^{2}, \mathrm{n} \neq \phi, \phi>1$;

b. $\mathrm{E}_{\mathrm{sen}}+\left(\mathrm{L}+\mathrm{N}^{2} \mathrm{~L}+\mathrm{L}^{2} / \theta\right) \mathrm{E}_{\mathrm{c}}+[2(\mathrm{~N}-1) \mathrm{ML}+4 \mathrm{~J}]$

$\mathrm{E}_{\mathrm{elec}}+\mathrm{ML} \epsilon_{\mathrm{fs}} \sum=1 \quad{ }_{1, \mathrm{n}}^{2}+4 \mathrm{~J} \epsilon_{\mathrm{fs}} \mathrm{d}_{1,2}^{2}, \mathrm{n} \neq 1$;

c. $\mathrm{E}_{\text {sen }}+\left(\mathrm{L}+\mathrm{N}^{2} \mathrm{~L}+\mathrm{L}^{2} / \theta\right) \mathrm{E}_{\mathrm{c}}+[2(\mathrm{~N}-1) \mathrm{ML}+4 \mathrm{~J}]$

$\mathrm{E}_{\text {elec }}+\mathrm{ML} \epsilon_{\mathrm{fs}} \sum=1 \quad 2 \quad{ }_{\phi, \mathrm{n}}, \mathrm{n} \neq \phi$;

d. $\mathrm{E}_{\mathrm{sen}}+2(\mathrm{~N}-1) \mathrm{MLE}_{\mathrm{elec}}+\mathrm{ML} \epsilon_{\mathrm{fs}} \sum=1 \quad{ }_{\mathrm{x}, \mathrm{n}}^{2}, \mathrm{x}=\theta+1 \ldots \mathrm{N}, \mathrm{n} \neq \mathrm{x}$.

\section{COMPARISON OF TOPOLOGIES ON THE BASIS OF ENERGY}

EFFICIENCY: Below are the results obtained from the comparison of total energy consumption in sensor networks:

If $\mathrm{M}(\mathrm{N}-2) \mathrm{E}_{\text {elec }}+\epsilon_{\mathrm{fs}} \mathrm{M}(\mathrm{N}-1) \mathrm{D}^{2} / 3+\left(\mathrm{N}^{2}+\mathrm{L}-1\right) \mathrm{E}_{\mathrm{c}}<\epsilon_{\mathrm{mp}} \mathrm{MN}\left(\mathrm{D}^{4} / 5+2 \mathrm{H}^{2} \mathrm{D} / 3+\mathrm{H}^{4}\right)$, then cluster based sensor network is more energy efficient than the sensor network with a fusion center and vice versa.

If $M\left(N^{2}-3 N\right) E_{\text {elec }}+2 \epsilon_{\mathrm{fs}} M N(N-1) D^{2} / 3+\left(\Theta N^{2}+L\right) E_{c}>\epsilon \quad M N\left(D^{4} / 5+2 H^{2} D^{2} / 3+H^{4}\right)$, 
International Journal of Distributed and Parallel Systems (IJDPS) Vol.3, No.5, September 2012

The concatenated sensor network is less energy efficient than the sensor network with a fusion center and vice versa.

6. COMPARISON OF THE TOPOLOGIES ON THE BASIS OF LIFETIME: When $\mathrm{N}$ is sufficiently large, the lower bound of $\mathrm{d}_{\mathrm{i} ; \mathrm{\beta}}^{2}$ and $\mathrm{d}_{\mathrm{i} ; \mathrm{n}}^{2}$ is zero and lower bound of $\mathrm{d}^{2}{ }_{\mathrm{i} ; \mathrm{f}}$ is $\mathrm{H}^{4}$.

In the sensor network with a fusion center, it is certain that $E_{m ; j}$ will approach $E_{s e n}+L_{c}+$

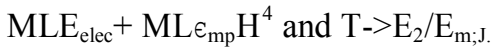

In the cluster-based sensor network, $\mathrm{d}_{\mathrm{J}-1 ; \beta}$ will approach zero. Because $(\mathrm{N}-2) \mathrm{ME}_{\text {elec }}+(\mathrm{N} 2+$ L-

1) $\mathrm{E}_{\mathrm{c}}>0$, we have $\mathrm{Eh}>\mathrm{E}_{\mathrm{m} ; \mathrm{J}-1 .}$. It is easy to verify that $\mathrm{T} \rightarrow \mathrm{E}_{1} / \mathrm{E}_{\mathrm{h}}$ if $\mathrm{E}_{1} / \mathrm{E}_{\mathrm{h}}<\mathrm{E}_{2} / \mathrm{E}_{\mathrm{m} ; \mathrm{J}-1}-1 ; \mathrm{E}_{\mathrm{m} ; \mathrm{J}-}$ ${ }_{1}$ approaches $\mathrm{E}_{\text {sen }}+\mathrm{LE}_{\mathrm{c}}+\mathrm{MLE}_{\text {elec }}$ and $\mathrm{T} \rightarrow \mathrm{E}_{2} / \mathrm{E}_{\mathrm{m}, \mathrm{J}-1}$ if $\mathrm{E}_{1} / \mathrm{E}_{\mathrm{h}}>\mathrm{E}_{2} / \mathrm{E}_{\mathrm{m} ; \mathrm{J}-1}+1$. Because the sensors are energy limited, $\mathrm{E}_{1} / \mathrm{E}_{\mathrm{h}}<\mathrm{E}_{2} / \mathrm{E}_{\mathrm{m} ; \mathrm{J}-1}-1$ is more likely than $\mathrm{E}_{1} / \mathrm{E}_{\mathrm{h}}>\mathrm{E}_{2} / \mathrm{E}_{\mathrm{m} ; \mathrm{J}-1}+1$.

The sensor network with a fusion center has longer lifetime than the concatenated sensor network. The comparative lifetime of the cluster-based sensor network to the sensor network with a fusion center and the concatenated sensor network dependsupon $E_{1} / E_{2}$, the system parameters and theenergy parameters.

\section{ENERGY EFFICIENCY OF THE TOPOLOGIES FOR THE GIVEN}

\section{SCENARIO}

Consider a grid of 10x10 as shown in the figure below. A sensor network can be arranged on this grid using a uniform distribution or random/Poisson distribution.

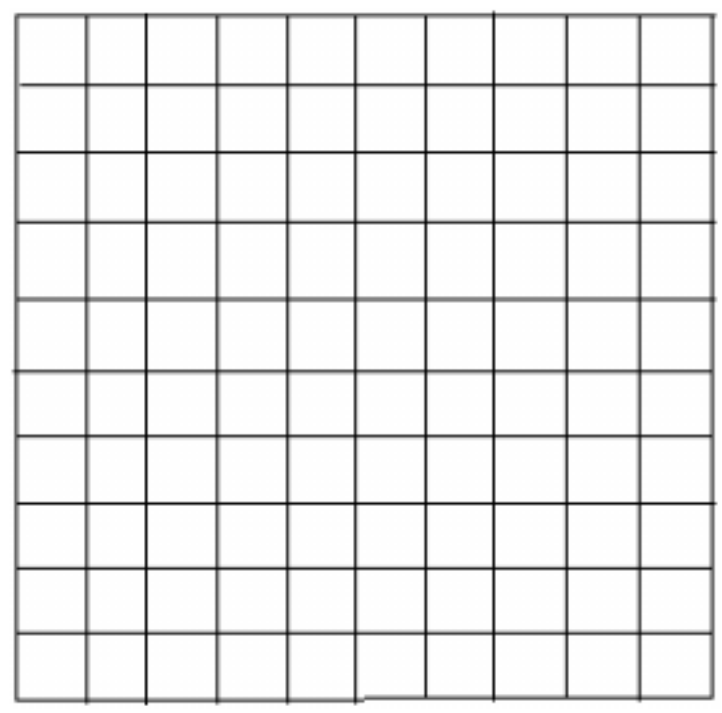

Fig.8 : 10x10 grid

The parameter values are given in the table below: 
International Journal of Distributed and Parallel Systems (IJDPS) Vol.3, No.5, September 2012

\begin{tabular}{|l|l|}
\hline \multicolumn{1}{|c|}{ Parameter } & \multicolumn{1}{|c|}{ Value } \\
\hline Energy consumption of a sensor, $\mathrm{E}_{\text {elec }}$ & $50 \mathrm{~nJ} / \mathrm{bit}$ \\
\hline Energy consumption of a sensor during sensing & $50 \mathrm{~nJ}$ \\
\hline Energy consumption of a sensor during computation & $5 \mathrm{~nJ}$ \\
\hline Initial energy of a sensor & $2 \mathrm{~nJ}$ \\
\hline No. of quantization bits,M & 4 \\
\hline Length of signal frame,L & 100 \\
\hline Amplification energy factor for fusion sensor based, $e_{\mathrm{mp}}$ & $.0031 \mathrm{pJ} / \mathrm{bit} / \mathrm{m}^{2}$ \\
\hline Amplifiction energy factor for cluster based, $\epsilon_{\mathrm{fs}}$ & $10 \mathrm{pJ} / \mathrm{bit} / \mathrm{m}^{2}$ \\
\hline No. of sensors,N & 100 \\
\hline Distance between two adjacent sensors, $\mathrm{d}_{\mathrm{i}, \mathrm{n}}$ & $1 \mathrm{~m}$ \\
\hline
\end{tabular}

7.1 Case 1: Uniform distribution:In uniform distribution, a sensor is present at each point of the grid. Now using this arrangement, we apply both cluster based and fusion center based approaches.

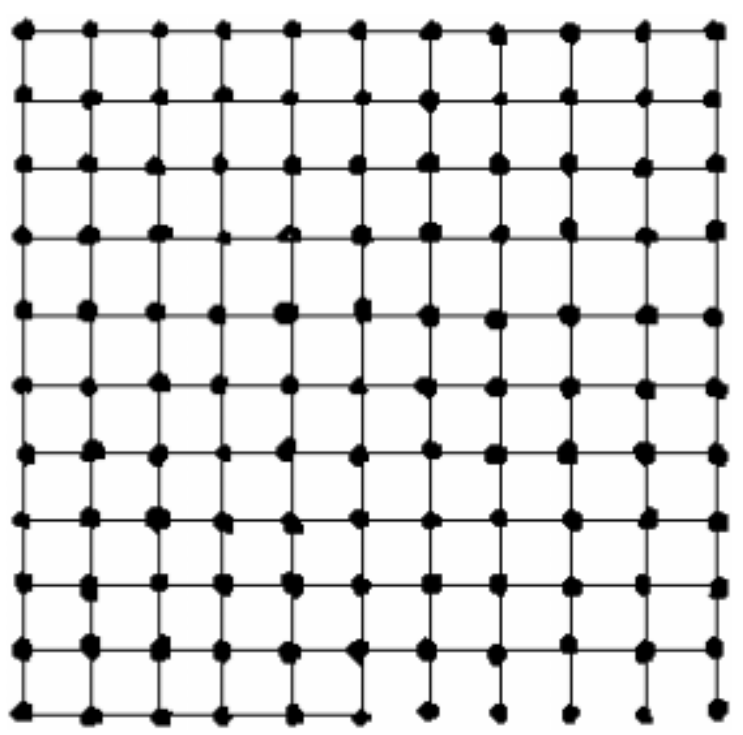

Fig.9: Sensors arranged in a uniform order in the grid. 
International Journal of Distributed and Parallel Systems (IJDPS) Vol.3, No.5, September 2012

7.1.1 Cluster based topology: Since in uniform distribution there are four identical clusters, we can calculate the energy of 1 cluster and multiply it by 4 .

$$
\begin{aligned}
& \mathrm{E}_{\text {comm. }}^{(1)}-2(\mathrm{~N}-1) \mathrm{MLE}_{\text {elec }}+M L \epsilon_{\mathrm{fs}} \sum=1 \quad{ }_{\mathrm{i}, \beta}^{2}, \mathrm{i} \neq \beta \\
& \text { i.e. } \mathrm{E}_{\text {comm. }}^{(1)}=1.4008 * 10^{-3} \mathrm{~J}
\end{aligned}
$$

Thus, for the whole system,

$$
\mathrm{E}_{\mathrm{comm}}=4 * 1.4008 * 10^{-3}=5.6032 * 10^{-3} \mathrm{~J}
$$

Energy consumed in computation,

$$
\mathrm{E}_{\text {comp }}=4 * 73980 * 10^{-9} \mathrm{~J}=2.959 * 10^{-4} \mathrm{~J}
$$

Energy consumed in sensing,

$$
\mathrm{E}_{\mathrm{sen}}=100 * 100 * 5 * 10^{-9} \mathrm{~J}=5 * 10^{-5} \mathrm{~J}
$$

Total energy consumption,

$\mathrm{E}_{\mathrm{total}}=560.32 * 10^{-5}+29.59 * 10^{-5}+5 * 10^{-5}=5.9491 * 10^{-3} \mathrm{~J}$

7.1.2 Fusion center based topology: For 1 part of the grid, energy consumed in communication is

$$
\mathrm{E}_{\text {comm. }}^{(1)}=36 * 4 * 100 * 50 * 10^{-9}+4 * 100 * 0.0013 * 10^{-12} * 134.88=7.2 * 10^{-4} \mathrm{~J}
$$

Total energy consumed in communication,

$\mathrm{E}_{\text {comm }}=4 * 7.2 * 10^{-4}=2.88 * 10^{-3} \mathrm{~J}$

Energy consumed in computation,

$$
\mathrm{E}_{\text {comp }}=100 * 100 * 5 * 10^{-9}=5 * 10^{-5} \mathrm{~J}
$$

Energy consumed in sensing,

$\mathrm{E}_{\mathrm{sen}}=100 * 100 * 5 * 10^{-9} \mathrm{~J}=5 * 10^{-5} \mathrm{~J}$

Total energy consumption,

$$
\mathrm{E}_{\text {total }}=288 * 10^{-5}+5 * 10^{-5}+5 * 10^{-5}=2.98 * 10^{-3} \mathrm{~J}
$$




\subsection{Case 2: Random Distribution}

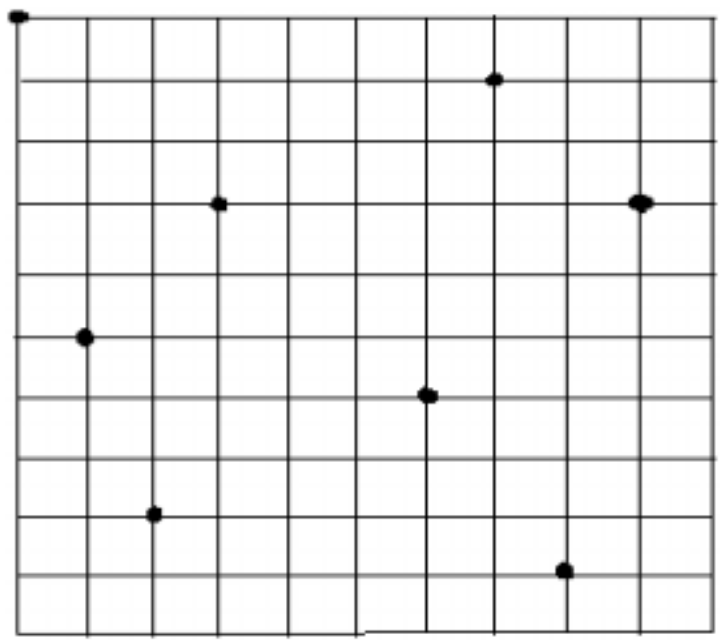

Fig.10: sensors arranged in a random order in the grid.

\subsubsection{Cluster based Topology:}

The total energy consumption in communication

$\mathrm{E}_{\mathrm{com}}=2 * 9 * 4 * 100 * 50 * 10^{-9}+4 * 100 * 10 * 10^{-12} * 53.380 \mathrm{~J}=3.602 * 10^{-4} \mathrm{~J}$

Total energy consumed in computation

$\mathrm{E}_{\text {comp }}=[9 * 100+100 * 4+16] * 5 * 10^{-9} \mathrm{~J}=6.58 * 10^{-6} \mathrm{~J}$

Total energy consumed in sensing,

$\mathrm{E}_{\mathrm{sen}}=10 * 100 * 5 * 10^{-5}=5 * 10^{-2} \mathrm{~J}$

Total energy consumption

$\mathrm{E}_{\text {total }}=360.2 * 10^{-6}+6.58 * 10^{-6}+50000 * 10^{-6}=5.0367 * 10^{-2} \mathrm{~J}$

\subsubsection{Fusion center based topology:}

The total energy consumed in communication,

$\mathrm{E}_{\mathrm{comm}}=10 * 4 * 100 * 50 * 10^{-9}+4 * 100 * .0013 * 10^{-12} * 3904.868 \mathrm{~J}=2.0 * 10^{-4} \mathrm{~J}$

The total energy consumed in computation,

$\mathrm{E}_{\text {comp }}=10 * 100 * 5 * 10^{-5}=5 * 10^{-2} \mathrm{~J}$

The total energy consumed in sensing,

$\mathrm{E}_{\mathrm{sen}}=10 * 100 * 5 * 10^{5}=5 * 10^{-2} \mathrm{~J}$

Total Energy Consuption,

$$
\mathrm{E}_{\text {total }}=.02 * 10^{-2}+5 * 10^{-2}+5 * 10^{-2}=.1 \mathrm{~J}
$$

From the above calculations, it can be seen that in uniform distribution of sensors, fusion center based topology consumes less energy as compared to the cluster based topology. On the other 
hand, when the distribution of the sensors is random, cluster based topology gives better results as compared to the fusion center based topology.

\section{Conclusion}

The presented paper provides a comparison of different topologies for the wireless sensor network. Cluster based topology and Fusion center based topology are used for the comparison. These topologies are compared on the basis of the two most important factors for a WSN i.e. lifetime and energy efficiency. From the above comparison, it is concluded that Fusion center based topology is more energy efficient in case of uniform distribution of sensors. On the other hand, when the sensors are distributed randomly, cluster based topology proves to be more efficient.

\section{RELATED WORK}

1. Comparative Study of Wireless Sensor Networks Energy-Efficient Topologies and Power Saver Protocols. Ewa Niewiadomska-Synkiewicz, Piotr Kwasniewski and Izabela Windyga. Journal of Telecommunications and Information Technology, March 2009.

This paper addresses the issue of controlled data transmission in wireless sensor networks due to limited power supply provider by batteries.

2. A Performance Comparison of Different Topologies for Wireless Sensor Networks. Shrestha, A. Technologies for Homeland Security, 2007 IEEE Conference on. Page(s): 280285. 16-17 may 2007.

This paper compares various network topologies using performance criteria such as scalability, data-latency, network life, energy consumption, reliability etc. with main focus on reliability comparison.

3. Developing Energy- Efficient Topologies and Routing for Wireless Sensor Networks. Hui Tian, Hong Shen and Teruo Matsuzawa. Network and Parallel Computing, Lecture Notes in Computer Science, 2005, Vol. 3779/2005, 461-469.

This paper discusses and compares performance measures of different patterned topologies for designing wireless sensor networks so that required area coverage and connectivity can be achieved. A proposal for several routing protocols is also made for wireless sensor networks in this paper.

\section{REFERENCES}

1. Impact of Topology on Performance and Energy Efficiency in Wireless Sensor Networks for SourceExtraction. Hongbin Chen, Chi K. Tse, and JiuchaoFeng. IEEE Transactions on Parallel AndDistributed Systems, Vol. 20, No. 6, June 2009

2. Strategies and techniques for node placement in wireless sensor networks: A survey. MohamedYounis, Kemal Akkaya. Adhoc Networks 6 (2008) 621-655

3. Handbook of Sensor Networks: Compact Wireless and Wired Sensing Systems CRC PRESS BocaRaton London New York Washington, D.C.

4. An Overview on Wireless Sensor Networks. Fabian Nack

5. G. Bell, "A time and a place for standards," Queue, vol. 2, no. 6, pp. 66-74, 2004.

6. M. W. Chiang, Z. Zilic, J.-S. Chenard, and K. Radecka, "Architectures of increased availabilitywireless sensor network nodes," Test Conference, International, vol. 0, pp. 1232-1241, 2004. 
International Journal of Distributed and Parallel Systems (IJDPS) Vol.3, No.5, September 2012

7. A. E. Kateeb, A. Ramesh, and L. Azzawi, "Wireless sensor nodes processor architecture and design", Advanced Information Networking and Applications Workshops, International Conference on, vol. 0,pp. 892-897, 2008.

8. K. S. Low, H. A. Nguyen, and H. Guo, "Optimization of sensor node locations in a wireless sensornetwork," International Conference on Natural Computation, vol. 5, pp. 286-290, 2008.

9. M. A. Taleghan, A. Taherkordi, M. Sharifi, and T.-H. Kim, "A survey of system software for wirelesssensor networks," Future Generation Communication and Networking, vol. 2, pp. 402 407, 2007 